\section{Synergistic activity of IDH1 inhibitor BAY1436032 with azacitidine in IDH1 mutant acute myeloid leukemia}

\author{
Anuhar Chaturvedi, ${ }^{1}$ Charu Gupta, ${ }^{1}$ Razif Gabdoulline, ${ }^{1}$ Nora M. Borchert, ${ }^{1}$ \\ Ramya Goparaju, ${ }^{1}$ Stefan Kaulfuss, ${ }^{2}$ Kerstin Görlich, ${ }^{1}$ Renate Schottmann, ${ }^{1}$ \\ Basem Othman, ${ }^{1}$ Julia Welzenbach, ${ }^{3}$ Olaf Panknin, ${ }^{2}$ Markus Wagner, ${ }^{2}$ \\ Robert Geffers, ${ }^{4}$ Arnold Ganser, ${ }^{1}$ Felicitas Thol, ${ }^{1}$ Michael Jeffers, ${ }^{5}$ \\ Andrea Haegebarth ${ }^{2}$ and Michael Heuser ${ }^{1}$
}

${ }^{1}$ Department of Hematology, Hemostasis, Oncology and Stem Cell Transplantation, Hannover Medical School, Hannover, Germany; ${ }^{2}$ Bayer AG, Berlin, Germany; ${ }^{3}$ Institute of Human Genetics, University of Bonn, School of Medicine \& University Hospital Bonn, Bonn, Germany; ${ }^{4}$ Genome Analytics Research Group, Helmholtz Center for Infection Research, Braunschweig, Germany and ${ }^{5}$ Bayer AG, Whippany, NJ, USA

\section{ABSTRACT}

M utant isocitrate dehydrogenase 1 ( $\mathrm{mIDH} 1)$ inhibitors have shown single-agent activity in relapsed/refractory acute myeloid leukemia (AML), even though most patients eventually relapse. We evaluated the efficacy and molecular mechanism of the combination treatment with azacitidine, which is currently the standard of care in older AML patients, and mIDH1 inhibitor BAY1436032. Both compounds were evaluated in vivo as single agents and in combination with sequential (azacitidine, followed by BAY1436032) or simultaneous application in two human IDH1 mutated AML xenograft models. Combination treatment significantly prolonged survival compared to single agent or control treatment $(P<0.005)$. The sequential combination treatment depleted leukemia stem cells by 470 -fold. Interestingly, the simultaneous combination treatment depleted leukemia stem cells by 33,150-fold compared to control mice. This strong synergy is mediated through inhibition of MAPK/ERK and Rb/E2F signaling. Our data strongly argues for the concurrent application of mIDH1 inhibitors and azacitidine and predicts improved outcome of this regimen in IDH1 mutated AML patients.

\section{Introduction}

In acute myeloid leukemia (AML) patients, mutated isocitrate dehydrogenase 1 (IDH1) is found in about $6-10 \%$ of patients ${ }^{1,2}$ at amino acid arginine 132 in the active site where isocitrate and NADPH bind. ${ }^{3}$ Whereas the enzymatic function of mutant IDH to convert isocitrate to $\alpha$-ketoglutarate is impaired, it gains a neomorphic function to convert $\alpha$-ketoglutarate to R-2-hydroxyglutarate (R-2HG), which induces histone- and DNA hypermethylation through inhibition of demethylation, and leads to a block in cellular differentiation thus promoting tumorigenesis. ${ }^{3-6} \mathrm{IDH} 1$ mutant-specific (mIDH1) inhibitors have emerged as a potent tool for the treatment of IDH1 mutant AML. A recent report on the first clinical IDH1 inhibitor ivosidenib as a single agent in IDH1-mutated relapsed or refractory AML showed an overall response rate of $41.6 \%$ and a complete remission rate of $21.6 \%$ with a median duration of response of 8.2 months. ${ }^{7}$ While these results are promising in this difficult to treat patient setting, they also suggest that $\mathrm{mIDH} 1$ inhibitors should be combined with other agents to improve efficacy.

A possible mechanism of synergy in IDH1 mutant AML is the reversal of DNA methylation by hypomethylating agents. 5-azacitidine (AZA) is a hypomethylating agent and can activate key epigenetically silenced pathways in AML cells, leading to an arrest of AML cell proliferation. ${ }^{8}$ The AZA-AML-001 study showed that azacitidine has clear activity in AML as monotherapy in patients with unfavorable cytogenetics. ${ }^{9}$ Therefore, a successful reversal of IDH1 mutant induced DNA hypermethylation in AML cells represents a window of opportunity to enhance the efficacy of mIDH1 inhibitors. We have previously shown that BAY1436032 is a highly effective
Haematologica 2021

Volume 106(2):565-573

\section{Correspondence:}

MICHAEL HEUSER

heuser.michael@mh-hannover.de

\section{ANUHAR CHATURVEDI}

chaturvedi.anuhar@mh-hannover.de

Received: September 9, 2019.

Accepted: March 26, 2020.

Pre-published: April 2, 2020.

https://doi.org/10.3324/haematol.2019.236992

(C)2021 Ferrata Storti Foundation

Material published in Haematologica is covered by copyright. All rights are reserved to the Ferrata Storti Foundation. Use of published material is allowed under the following terms and conditions:

https://creativecommons.org/licenses/by-nc/4.0/legalcode. Copies of published material are allowed for personal or internal use. Sharing published material for non-commercial purposes is subject to the following conditions: https://creativecommons.org/licenses/by-nc/4.0/legalcode, sect. 3. Reproducing and sharing published material for commercial purposes is not allowed without permission in writing from the publisher. 
oral pan-mutant IDH1 inhibitor, which has strong antileukemic activity in patient-derived xenograft (PDX) models of IDH1 mutant leukemia in vivo by inducing differentiation of leukemic blasts as well as inhibition of leukemia stem cell proliferation and self-renewal. ${ }^{10}$ BAY1436032 is currently evaluated in phase I clinical trials in AML and glioma patients (NCT03127735 and NCT02746081).

In this study, we evaluated the therapeutic efficacy and elucidated the mechanism of action of the combined treatment of azacitidine and BAY1436032 in IDH1 mutant AML in vitro as well as in vivo in two independent patient-derived xenograft models of IDH1 mutant AML. From these experiments, we conclude that the simultaneous combination of a $\mathrm{mIDH}$ inhibitor with a hypomethylating agent synergistically inhibits LSC through suppression of MAP kinase and $\mathrm{RB} / \mathrm{E} 2 \mathrm{~F}$ signaling, which are involved in cell survival and proliferation.

\section{Methods}

\section{Combination index}

Drug synergy was evaluated using a combination index (CI) equation based on the multiple drug-effect equation of ChouTalalay. ${ }^{11,12}$ Colony-forming cell units were assayed in methylcellulose after treatment with varying doses of either azacitidine (62.5, $125,250,500,1,000$ or $2,000 \mathrm{~nm})$, BAY 1436032 ( $6.25,12.5,25$, 50,100 or $200 \mathrm{~nm}$ ) or a fixed ratio of the combination of azacitidine/BAY1436032 (62.5/6.25, 125/12.5, 250/25, 500/50, 1,000/100 or $2,000 / 200 \mathrm{~nm})$. The analysis was performed with CompuSyn software (ComboSyn Inc., Paramus, USA).

\section{Patient samples}

Diagnostic bone marrow or peripheral blood collected from AML patients at Hannover Medical School were analyzed for mutations in IDH1 and IDH2 by Sanger sequencing. Details of the type of IDH mutation are described in the figure legends. Mononuclear cells were isolated by ficoll density centrifugation, washed with PBS, and red blood cells were lysed using a red blood cell (RBC) lysis buffer (BD Pharm Lyse, BD Biosciences, Heidelberg, Germany). The bone marrow samples for the development of the PDX models were collected prior to the start of AML treatment. Written informed consent was obtained according to the Declaration of Helsinki, and the study was approved by the Institutional Review Board of Hannover Medical School, Hannover, Germany.

\section{Transplantation and treatment}

Six to eight-week old female NOD.Cg-Prkd scid $I l 2 \mathrm{rg}^{\text {tm } 1 \mathrm{Wi}} \mathrm{l} / \mathrm{SzJ}$ (NSG) mice were purchased from Hannover Medical School, Germany and kept in pathogen free conditions at the central animal laboratory of Hannover Medical School. Experimental procedures were approved by the governmental authorities of Lower Saxony, Germany, and supervised by local animal welfare officials. The IDH1 mutant AML PDX model was developed as described. ${ }^{10}$ One million patient-derived AML cells (hCD45+) were collected from the bone marrow and spleen of leukemic mice and were injected intravenously in the tail vein of sublethally (3 Gy) irradiated NSG mice. Neither randomization, nor blinding was used since all animal experiments were performed with a homogeneous strain, comparable age, and similar variance of the mice. Treatment was started 28 days after transplantation. The control groups were treated with either vehicle, BAY1436032 $150 \mathrm{mg} / \mathrm{kg}$ once daily orally continuously, or azacitidine $1 \mathrm{mg} / \mathrm{kg}$ once daily subcutaneoulsy on days $1-5$, repeated once after 28 days. The test groups were treated with BAY1436032 and azacitidine in the doses mentioned above either starting both drugs on day 1 (simultaneous group) or starting azacitidine on day 1 but BAY1436032 on day 6 (sequential group). In the second cycle in the sequential treatment group, BAY1436032 was stopped during azacitidine treatment to better model the biologic and therapeutic effects of the synergy. The treatment was stopped at day 84 or day 96 . The proportion of leukemic cells in the peripheral blood of mice was measured with the human-specific CD 45 antibody every 4 weeks by tail vein bleeds and fluorescence-activated cell sorting (FACS) analysis. ${ }^{13}$ Blood counts were performed using an ABC Vet Automated Blood counter (Scil animal care company $\mathrm{GmbH}$, Viernheim, Germany). All animal experiments were started with 10 animals, however, the animals which died before the start of treatment due to engraftment failure, during injections or during bleeding were excluded from the study.

\section{Statistical analysis}

Two-tailed unpaired comparisons were performed by MannWhitney test in Graph pad prism 8.2.1 (GraphPad Software, La Jolla, CA). Comparison of survival curves were performed using the log-rank test. Statistical analyses were performed with Microsoft Excel 2016 (Microsoft, Munich, Germany) or GraphPad Prism 8.2.1 (GraphPad Software, La Jolla, CA, USA). Graphical representation was prepared using Adobe Illustrator CS6 (Adobe systems $\mathrm{GmbH}$, Munich, Germany). The size of the animal cohorts was based on our previous study. ${ }^{5,6,10}$ All in vitro experiments were performed at least three times and all attempts of replication were successful.

\section{Results}

\section{mIDH1 inhibitor BAY1436032 and azacitidine synergize to inhibit human IDH1 mutant acute myeloid leukemia cells ex vivo}

In order to test the effect of the combination of BAY1436032 and azacitidine on survival and proliferation of primary human AML cells, IDH1 wild-type $(n=6)$ and IDH1 mutant $(\mathrm{n}=6)$ cells from AML patients were seeded in semi-solid medium supplemented without or with BAY1436032 in combination with varying concentrations of azacitidine. BAY1436032 reduced colony formation specifically in human IDH1 mutant AML cells with an IC of $100 \mathrm{nM}$, while IDH1 wild-type cells were not affected (Online Supplementary Figure S1A). Azacitidine alone induced a $20 \%$ reduction in colony formation in both IDH1 wild-type and IDH1 wild-type AML patient cells at $100 \mathrm{nM}$ (Online Supplementary Figure S1B), while the combination of BAY1436032 with azacitidine reduced colony formation in a dose-dependent manner in IDH1 mutant cells with reduction of colonies by $80 \%$ in IDH1 mutant AML but only by $20 \%$ in IDH1 wild-type AML cells at $100 \mathrm{nM}$ azacitidine, suggesting improved efficacy of an IDH1 inhibitor in combination with azacitidine (Figure 1A, normalized to cells treated with BAY1436032 at $100 \mathrm{nM}$ ). In order to test the effect on cell cycle progression and apoptosis, IDH1 mutant and IDH1 wild-type AML cells were cultured in suspension medium and treated with vehicle, $100 \mathrm{nM}$ BAY1436032 or $100 \mathrm{nM}$ azacitidine as single agents or in combination. The proportion of cells in S phase of the cell cycle was strongly decreased by the combination treatment compared with either monotherapy or vehicle in IDH1 mutant AML cells, however, remained largely unchanged in IDH1 wild-type cells (Figure 1B-C). No difference was 
observed in the proportion of G0/G1 cells between the combination and monotherapy groups, while the subG1 population was increased in the combination treatment group indicating increased cell death. The percentage of Annexin $\mathrm{V}^{+}$cells did not differ between any of the treatment groups in IDH1 wild-type or IDH1 mutant AML cells (Online Supplementary Figure S2). In order to further investigate the synergy between azacitidine and BAY1436032, we treated IDH1 mutant AML cells from six patients with serial dilutions of azacitidine and BAY1436032, either alone or in combination and performed colony forming unit assays. The combination of azacitidine with BAY14360932 significantly decreased the colony formation of cells treated with both agents (Figure 1D). In order to determine whether the combined effects of azacitidine and BAY1436032 are additive or synergistic, we performed an isobologram analysis from an average of six patients. The combination of azacitidine with BAY1436032 proved highly synergistic with a combination index (CI) of $<0.68$ at an effective dose (ED) of 95, a CI of $<0.65$ at ED 75, and a CI of $<0.63$ at ED 50, respectively (Figure 1E; a CI of 1 reflects an additive effect, a CI $<1$ a synergistic effect and a CI $>1$ indicates antagonism). This data suggest that BAY1436032 in combination with azacitidine more effectively inhibits proliferation of primary IDH1 mutant AML cells ex vivo than single agent treatment.

\section{BAY1436032 synergizes with azacitidine to exert potent} anti-leukemic activity in the patient-derived IDH1 mutant acute myeloid leukemia xenograft models in vivo

We have previously developed and characterized an IDH1 mutant PDX mouse model (AML-PDX1) using primary AML cells from a patient harboring IDH1 R132C, FLT3-TKD (p.D835del), an atypical NPM1 (p.S254LfsTer4), and a NRAS (p.Q61R) mutation. ${ }^{10}$ After 28 days of transplantation, having confirmed engraftment between $2-5 \%$ in peripheral blood, IDH1 mutant PDX mice were treated with either vehicle, BAY1436032, two cycles of azacitidine as monotherapy or in sequential or simultaneous combination of both drugs for a total duration of 84 days (Figure 2A). We evaluated the simultaneous and sequential combination treatment to derive mechanistic insight into the combination treatment and to instruct the clinical use of this combination, as mutation analysis usually takes some time and leaves a time window in which treatment with azacitidine may be started before the mutation status is known, followed by sequential treatment with a mIDH1 inhibitor.

While the engraftment of human leukemic cells increased in vehicle and azacitidine treated mice at week 8 after the start of treatment, the percentage of leukemic cells decreased in BAY1436032 treated mice as well as in the groups receiving the sequential and simultaneous combination treatment (Figure 2B). However, after the stop of treatment at week 12 the percentage of leukemic cells increased after week 16 in the treatment groups receiving BAY1436032 or the sequential combination of BAY1436032 and azacitidine (Figure $2 \mathrm{~B}$ ). Interestingly, the percentage of leukemic cells in mice treated with the simultaneous combination of BAY1436032 and azacitidine showed a delayed increase of blasts and slower kinetics (Figure 2B) and 2 of 6 mice from this cohort remained negative in peripheral blood until the end of the study at 36 weeks (Figure 2C). The mean IDH1 mutant allele fraction in peripheral blood at 8 weeks after the stop of the treatment was $48.8 \%$ in vehicle, $40.56 \%$ in the azacitidine, $46.43 \%$ in the BAY1436032, and $43.2 \%$ in the sequential treatment cohorts. However, median IDH1 mutant allele burden was $10.89 \%$ in the simultaneous treatment cohort, and below the detection limit in 3 of 5 analysed mice (Online Supplementary Figure S3A). While the white blood cell counts constantly increased and platelet counts, as well as hemoglobin, decreased in mice receiving vehicle, azacitidine or BAY1436032 monotherapy or the sequential combination of BAY1436032 and azacitidine, all mice treated simultaneously had normal blood counts until week 36 (Figure 2D-E, Online Supplementary Figure S3B). In order to compare the effects of the combination therapy with single agents on the induction of myelomonocytic differentiation the expression of CD14 and CD15 in human CD45 cells was monitored. At day 30 after initiation of treatment there was no significant difference in the expression of the myelomonocytic maturation markers CD14 and CD15 in the vehicle (CD14 0.05 \pm 0.05 ; CD15 0.86 \pm 0.3 ) and azaciti-

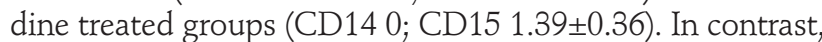
mice treated with BAY1436032 had a significantly higher proportion of cells expressing CD14 (4.21 \pm 0.62$)$ and CD15 (7.67 \pm 0.68$)$. Mice treated in parallel with the combination of BAY1436032 and azacitidine had additive effects on the expression of these markers (CD14 7.44 1.27 ; CD15 $11 \pm 0.79$ ) (Online Supplementary Figure S4). While azacitidine treated mice survived longer with a median survival of 188 days compared to vehicle-treated mice with a median survival of 139 days, BAY1436032 treated mice had significantly longer latency with a median survival of 220 days. However, mice treated sequentially with the combination of BAY1436032 and azacitidine survived longer than with BAY1436032 monotherapy with a median survival of 299 days. Strikingly, 5 of 6 mice treated simultaneously with BAY1436032 and azacitidine survived until the end of the study at 300 days and the median survival was not reached (Figure 2F).

In an independent, second PDX model (AML-PDX2), which harbored IDH1 p.R132H, DNMT3A p.R882H, PTPN11 p.A72T, and NPM1 p.T288CEsTer12 mutations (Online Supplementary Figure S5A), the percentage of human CD $45^{+}$cells increased in vehicle, azacitidine, BAY1436032 and sequential combination treatment groups albeit with different kinetics (Online Supplementary Figure S5B). However, even after the stop of treatment at week 12, the percentage of leukemic cells in mice treated with the simultaneous combination of BAY1436032 and azacitidine remained low and 2 of 6 mice from this cohort had less than $10 \%$ human $\mathrm{CD} 45^{+}$cells in the peripheral blood until the end of the study at 28 weeks (Online Supplementary Figure $S 5$ C). In contrast to the other groups, all mice treated simultaneously had normal blood counts until week 16 after the start of treatment (Online Supplementary Figure S5D-F). At day 90 after initiation of treatment mice treated simultaneously with the combination of BAY1436032 and azacitidine had significantly higher expression of CD15 than mice treated with BAY1436032 alone or with the sequential combination of BAY1436032 and azacitidine (Online Supplementary Figure S5G). The mice treated simultaneously with BAY1436032 and azacitidine survived significantly longer with a median survival of 250 days than sequentially treated mice with a median median survival of 186 days (Online Supplementary Figure S5G).

In summary, the simultaneous combination of 
A

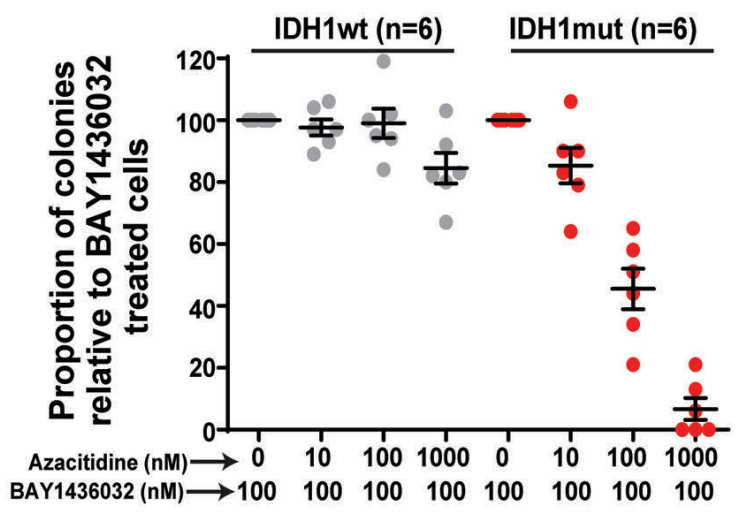

B

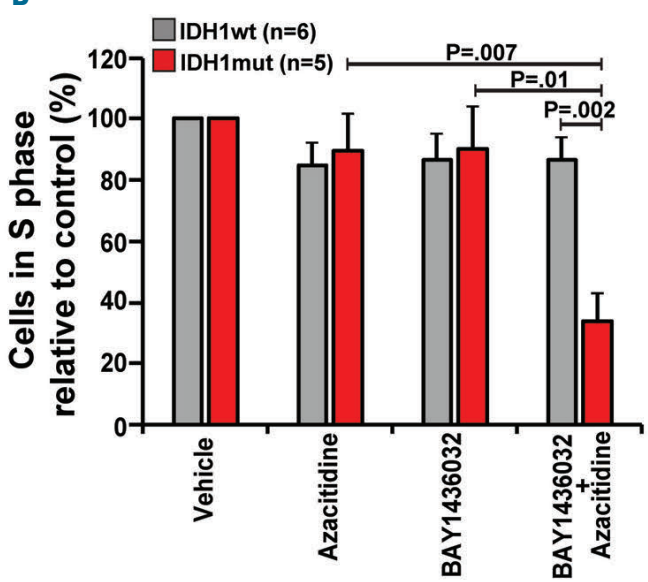

C
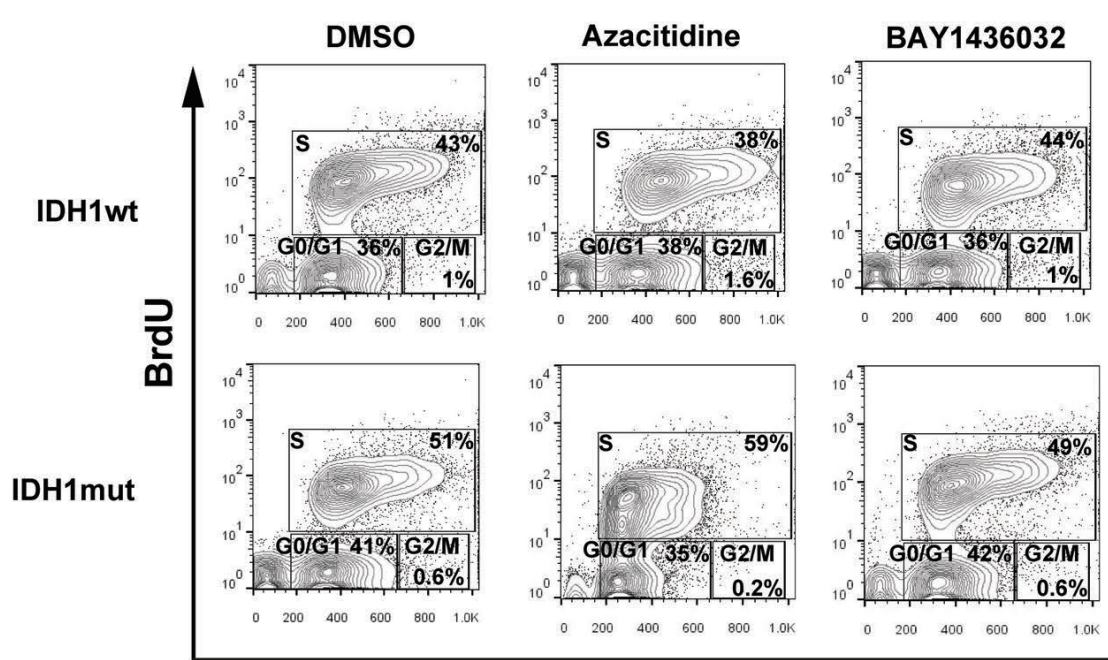

BAY1436032

Azacitidine
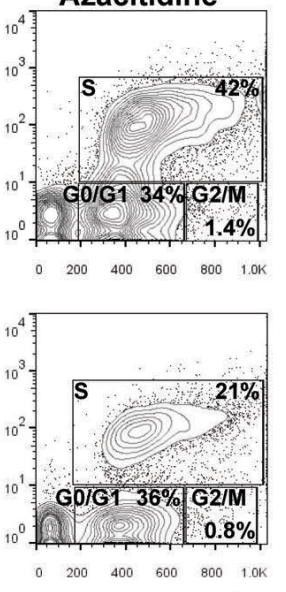

7-AAD

D

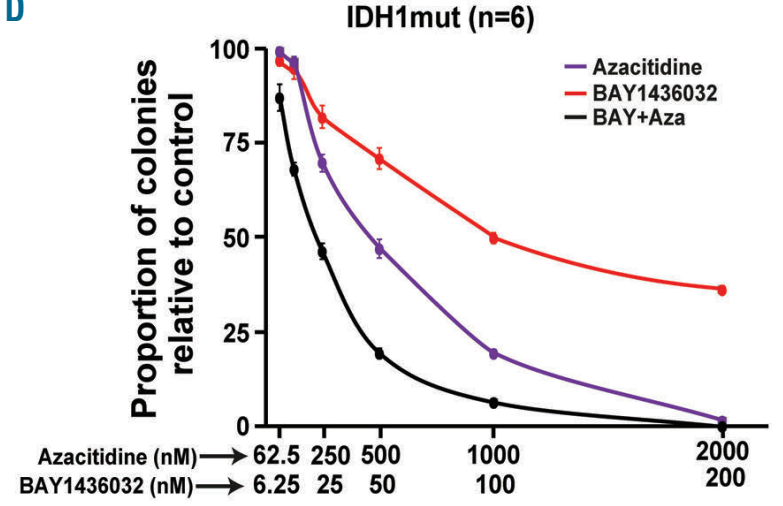

E

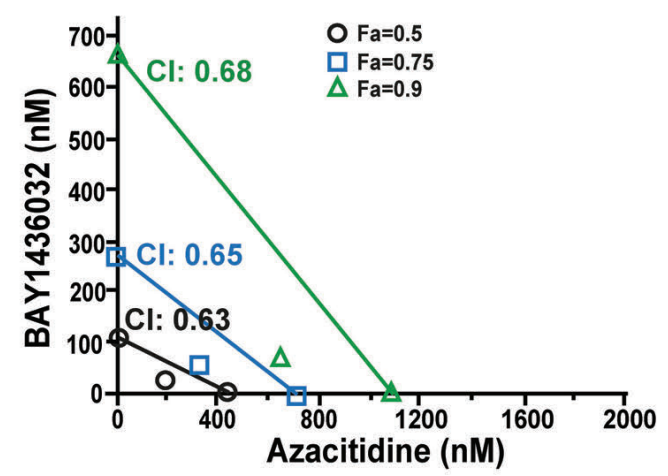

Figure 1. Mutant IDH1 inhibitor BAY1436032 and azacitidine synergize to inhibit human IDH1 mutant acute myeloid leukemia cells ex vivo. (A) Inhibition of colony formation by combining BAY1436032 (100 nm, corresponding to the $I_{\mathrm{s}}$ in previous experiments) with varying concentrations of azacitidine in colony forming assays using primary human acute myeloid leukemia (AML) cells with wild-type or mutant IDH1. The graph represents the proportion of colonies relative to cells treated with BAY1436032 at $100 \mathrm{nM}$ (mean \pm standard error of the mean). From the six patients with IDH1 mutant AML, four harbored an IDH1 R132H mutation and one each an IDH1 R132C and IDH1 R132G mutation. (B) Proportion of viable cells in S phase of the cell cycle after treatment with BAY1436032 (100 nM) or azacitidine (100 $\mathrm{nM}$ ) or the combination of both relative to DMSO-treated cells (mean \pm standard error of the mean). From the 5 patients with IDH1 mutant AML, 3 harbored an IDH1 $\mathrm{R} 132 \mathrm{H}$ mutation and 1 each an IDH1 R132C and IDH1 R132G. (C) A representative fluorescence-activated cell sorting plot of IDH1 wild-type and IDH mutant primary AML cells treated ex vivo with either vehicle, BAY1436032, azacitidine or BAY1436032 and azacitidine in combination. (D) Inhibition of colony formation after treatment with serial dilutions of azacitidine and BAY1436032, alone or in combination using primary human IDH1 mutant AML cells. Five patients harbored an IDH1 $\mathrm{R} 132 \mathrm{H}$ and one an IDH1 R132C mutation. (E) Isobologram analysis of the combination of azacitidine and BAY1436032 in IDH1 mutant AML patient cells. The individual doses of azacitidine and BAY1436032 to achieve 90\% growth inhibition (effective dose [ED] or fraction affected [Fa]=0.9), $75 \%$ growth inhibition (ED 75 or $\mathrm{Fa}=0.75$ ), and $50 \%$ growth inhibition (ED 50 or $\mathrm{Fa}=0.5$ ) were plotted on the $\mathrm{x}$ - and $\mathrm{y}$-axes. Combination index $(\mathrm{Cl})$ values calculated using CompuSyn software is depicted in the graph. $\mathrm{A} \mathrm{Cl}$ of 1 indicates an additive effect, a $\mathrm{Cl}<1$ a synergistic effect and a $\mathrm{Cl}>1$ antagonism. Wt: wild-type, mut: mutant. 
A

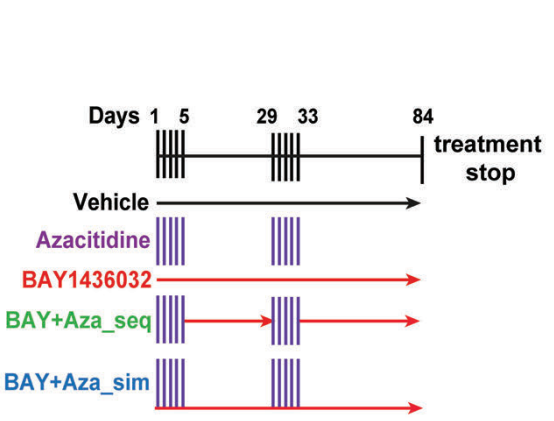

D

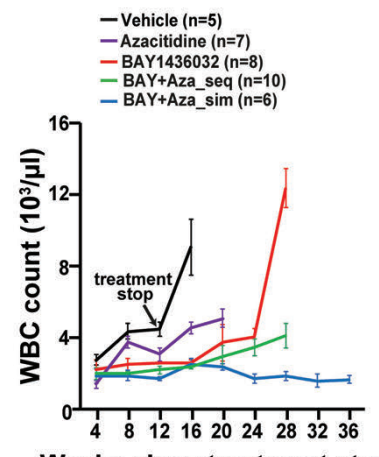

Weeks since treatment start

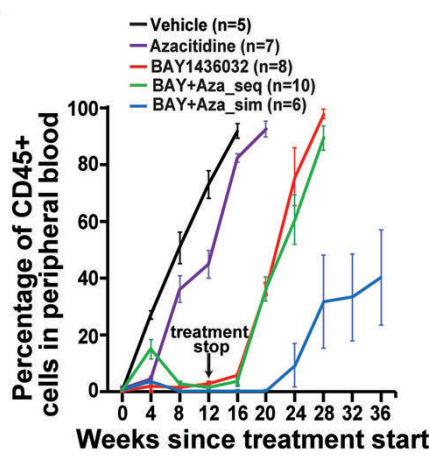

$E$

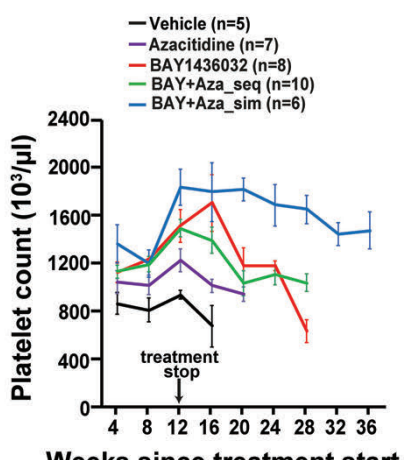

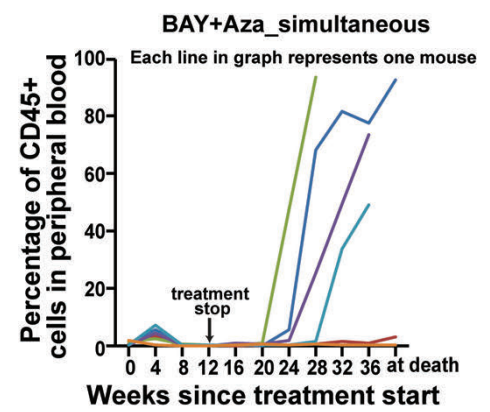

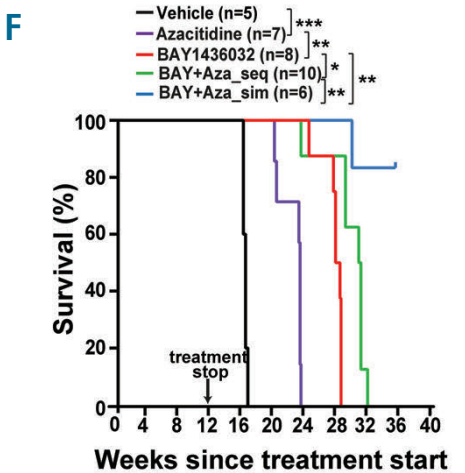

Figure 2. BAY1436032 synergizes with azacitidine to exert potent anti-leukemic activity in a patient-derived IDH1 mutant acute myeloid leukemia xenograft model in vivo. (A) Schematic representation of the treatment regimens; sim: simultaneous treatment with BAY1436032 and azacitidine (Aza); seq: sequential treatment with BAY1436032 and azacitidine. (B) Percentage of hCD45+ leukemic cells in peripheral blood of IDH1 R132C PDX1 mice at different time points after treatment start with vehicle, azacitidine (1 mg/kg, subcutaneoulsy, days $1-5$ and days 29-34), BAY1436032 (150 mg/kg orally, continuously), or the sequential or simultaneous combination of BAY1436032 and azacitidine according to the treatment regimen shown in Figure $2 \mathrm{~A}$ (mean \pm standard error of the mean [SEM]). (C) Percentage of hCD45+ leukemic cells in peripheral blood of individual mice transplanted with human IDH1 mutant AML cells and simultaneously treated with BAY1436032 and azacitidine. (D) White blood cell counts after different time points of treatment (mean \pm SEM). (E) Platelet counts after different time points of treatment (mean \pm SEM). (F) Kaplan-Meier survival curves of IDH1 mutant PDX1 mice treated with vehicle, azacitidine (1 mg/kg, subcutaneoulsy), BAY1436032 (150 mg/kg, orally), or the sequential or simultaneous combination of BAY1436032 and azacitidine according to the treatment regimen shown in Figure $2 \mathrm{~A}$. $* P<0.5$; $* * P<0.01$; $* * * P<0.001$; PDX: patient-derived xenograft; wt: wild-type; mut: mutant.

BAY1436032 and azacitidine is highly effective in inhibiting IDH1 mutated human leukemia in vivo, suggesting a specific dependency on the simultaneous presence of both drugs in vivo.

\section{Combination treatment with BAY1436032 and} azacitidine strongly depletes leukemia stem cells in vivo through inhibition of MAP-kinase signaling and activation of myeloid differentiation

In order to assess the effect of simultaneous and sequential treatment with BAY1436032 and azacitidine on leukemia stem cell self-renewal we performed a limiting dilution transplantation experiment with IDH1 mutant AML cells. NSG mice transplanted with primary human IDH1R132C mutant AML cells were treated when leukemias were fully established $(70-80 \%$ human AML cells in the peripheral blood) with either vehicle, azacitidine, BAY1436032, or the sequential or simultaneous combination of BAY1436032 and azacitidine for 4 weeks (Online Supplementary Figure S6A). Subsequently, we harvested bone marrow cells from these treatment groups and transplanted defined numbers of human AML cells in recipient mice at limiting dilution. Cell numbers of 2,000,000, 200,000, 20,000, 2,000, 200, and 20 human CD45 AML cells were injected into three NSG recipient mice per cell dose for each treatment group. After 8 weeks the mice were bled and scored positive if more than $0.1 \%$ human $\mathrm{CD} 45^{+}$ cells were present in the peripheral blood (Figure 3A). The LSC frequency was 1 in 73 in vehicle-treated mice, 4.1-fold lower in azacitidine treated mice (1 in 304), and 117-fold lower in BAY1436032 treated mice (1 in 8,580). The sequential combination treatment of BAY1436032 and azacitidine decreased the stem cell frequency by 470 -fold (1 in 34,300) compared to control treated mice and by 4-fold compared to BAY1436032 monotherapy. However, in mice treated simultaneously with BAY1436032 and azacitidine, the leukemia stem cell frequency was 33,150-fold lower compared to control treated mice, 282-fold lower compared to BAY1436032 treated mice, and 70-fold lower compared to mice treated sequentially with BAY1436032 and azacitidine (1 in 2,420,000, Figure 3A, Online Supplementary Figure 6B).

Next, we performed gene expression profiling of $\mathrm{hCD}^{+} 5^{+}$ cells from NSG mice treated with vehicle, azacitidine, BAY1436032 or simultaneous treatment with BAY1436032 and azacitidine for 4 weeks. In unsupervised hierarchical clustering, azacitidine and vehicle-treated groups clustered together and separated from BAY1436032 and BAY1436032 + azacitidine treated groups. However, many genes that were overexpressed in BAY1436032 treated cells were suppressed in the combination group (Figure 3B). Principal component analysis showed that BAY1436032 + azacitidine treated cells separated well from the control or singleagent treated cells (Figure 3C). Interestingly, 11 of 17 genes from the LSC17 gene signature were downregulated by the 
A
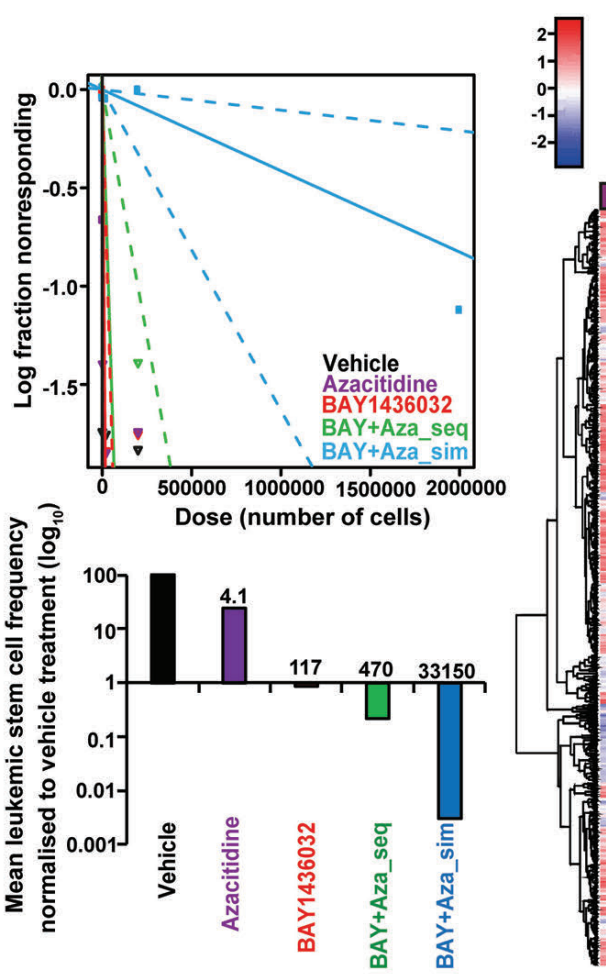

E

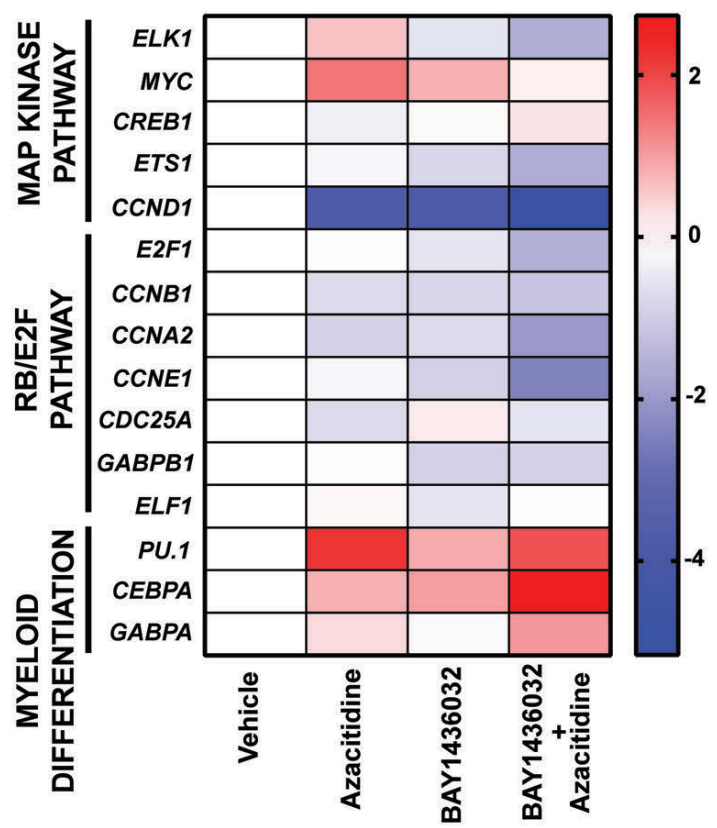

C

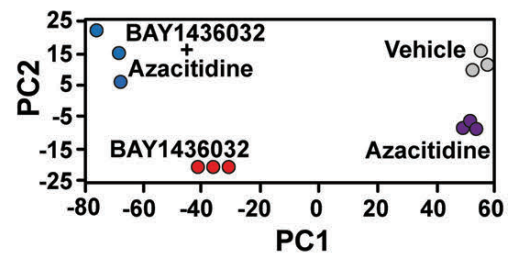

D

GAzacitidine vs Vehicle
BAY1436032 vs Vehicle
BAY+Aza vs BAY Aza Vehicle

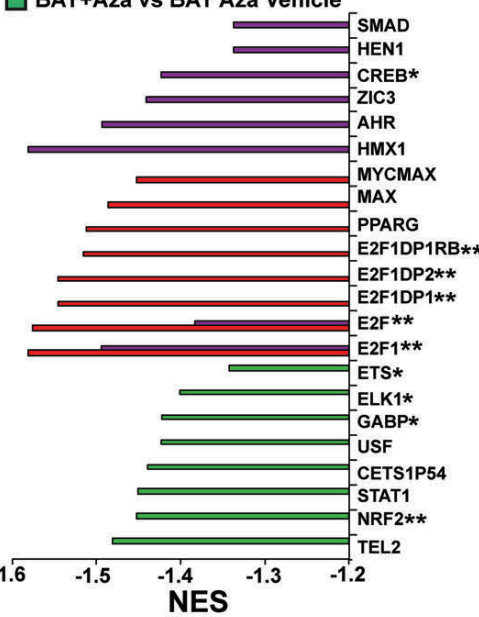

$\mathrm{F}$

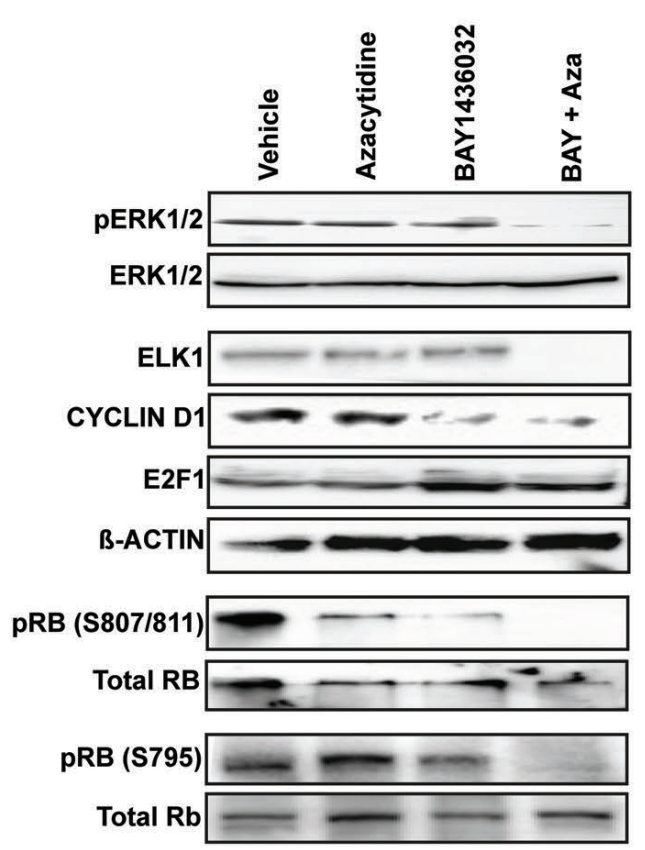

G

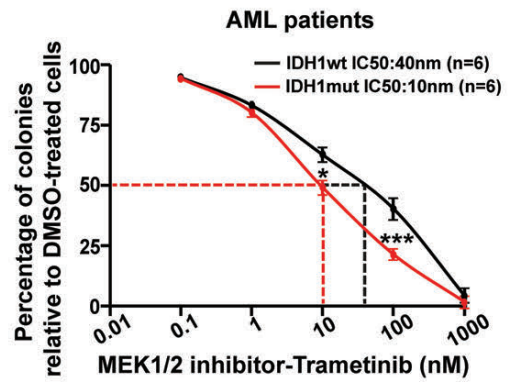

H

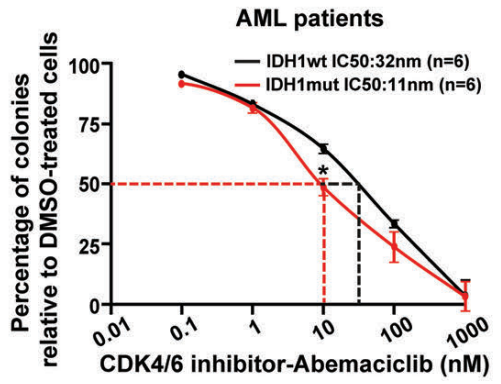


Figure 3 on previous page. Combination treatment with BAY1436032 and azacitidine strongly depletes leukemia stem cells in vivo through inhibition of MAPkinase signaling and activation of myeloid differentiation. (A) Limiting dilution transplantation of bone marrow cells from IDH1 mutant patient-derived xenograft (PDX) mice treated with vehicle, azacitidine (1 mg/kg, subcutaneously, days 1-5), BAY1436032 (150 mg/kg, orally [p.o.], daily [q.d.], 4 weeks) or the sequential or simultaneous combination of BAY 1436032 and azacitidine with the same doses as in the single agent treated mice. 2,000,000, 200,000, 20,000, 2,000, 200 or 20 human acute myeloid leukemia (AML) cells per mouse were transplanted into three recipient mice per cell dose. Leukemia stem cells (LSC) frequencies are shown (mean \pm standard error of the mean [SEM], $n=3$ ). Mice with hCD45+ cells in peripheral blood after 8 weeks $(>0.1 \%)$ were scored positive. The log-fraction plot of the limiting dilution model has been generated using the ELDA software; the median LSC frequencies are shown by solid lines and the $95 \%$ confidence intervals $(\mathrm{Cl})$ by dotted lines. The fold change in LSC frequency normalized to vehicle treated control mice is shown as a bar graph. The fold decrease in LSC compared to vehicle treated mice is indicated on top of the bars. (B) Unsupervised hierarchical clustering using euclidean distance of cells from bone marrow of IDH1 mutant PDX mice treated with vehicle, azacitidine (1 mg/kg, subcutaneously, days 1-5), BAY1436032 (150 mg/kg, orally [p.o.], daily [q.d.], for 4 weeks) or the simultaneous combination of BAY1436032 and azacitidine. Cells were harvested from bone marrow at 4 weeks after treatment and sorted for hCD45 ${ }^{+}$cells. Gene expression profiling using RNA was performed on Affymetrix Human HG_U133 Plus 2.0 microarrays ( $n=3$ per group). (C) Principal component analysis of all treatment groups using the top 4,000 differentially expressed genes. (D) Gene set enrichment analysis (MSigDB version 6.0) showing the most enriched transcription factor target gene sets from the indicated treatment comparisons. NES: normalized enrichment score; * gene sets involved in MAP Kinase signaling; ** gene sets involved in RB/E2F signaling (E) Heatmap from gene expression levels of MAP kinase signaling genes, RB/E2F signaling genes and myeloid differentiation genes from the bone marrow of IDH1 mutant PDX1 mice treated with vehicle, azacitidine (1 mg/kg, subcutaneous [s.c.] sq, days 1-5), BAY1436032 (150 mg/kg, p.o., q.d., 4 weeks) or the simultaneous combination of BAY1436032 and azacitidine. Gene expression was determined by quantitative RT-PCR relative to the housekeeping gene ABL and was normalized to gene expression in vehicle-treated cells (mean $+\mathrm{SEM}, \mathrm{n}=3$ independent experiments). ( $\mathrm{F}$ ) Representative western blots of in vitro cultured $\mathrm{HT} 1080$, a fibrosarcoma cell line with an endogenous heterozygous IDH1 R132C mutation treated with vehicle, azacitidine, BAY1436032 or the simultaneous combination of BAY1436032 and azacitidine using antibodies against the indicated signaling proteins. (G) Inhibition of colony formation by the MEK1/2 inhibitor trametinib in colony-forming cell assays using primary human AML cells with wild-type IDH1 or mutant IDH1 (mean \pm SEM). (H) Inhibition of colony formation by the CDK4/6 inhibitor abemaciclib in colony-forming cell assays using primary human AML cells IDH1 wild-type or IDH1 mutant (mean \pm SEM). From the six patients with IDH1 mutant AML, three harbored a IDH1 R132H mutation and one each an IDH1 R132C, IDH1 R132L and IDH1 R132G mutation. *P<0.5; ***P<0.001, wt: wild-type, mut: mutant: AZA: azacitidine.

combination treatment, supporting the inhibitory effect of the combination therapy on LSC (Online Supplementary Figure S7). ${ }^{14}$ Gene set enrichment analysis revealed that the suppressed transcription factors in the combination treatment group were linked to MAP kinase and retinoblastoma/E2F (Rb/E2F) signaling (Figure 3D, Online Supplementary Table S1-3). We also performed epigenome wide asscociation studies by DNA methylation arrays and compared them with the transcriptome obtained by RNA sequencing (RNASeq) in IDH1 R132C cells isolated from AML-PDX1 mice and treated in vitro with either DMSO, $500 \mathrm{nM}$ azacitidine (day 1-4), $50 \mathrm{nM}$ of BAY1436032 (day 1-5), and sequential or simultaneous treatments with BAY1436032 and azacitidine. Principal component analysis showed that sequentially treated cells separated well from simultaneously treated cells (Online Supplementary Figure $S 8 A-B)$. The simultaneous treatment induced strong synergistic gene expression ( $\mathrm{S}$-value $>0$ ), while the gene expression was mostly antagonistic in the sequential treatment group (S-value <0; Online Supplementary Figure S8C). The simultaneous treatment overall led to increased methylation compared to sequential treatment (Online Supplementary Figure S8D). Gene set enrichment analysis revealed that the suppressed transcription factors in the simultaneous treatment group were linked to MAP kinase signaling (RAS/RAF), while transcription factors enriched in single treatments were linked to $\mathrm{Rb} / \mathrm{E} 2 \mathrm{~F}$ signaling (Online Supplementary Figure 8E). In concordance with gene expression, an epigenome-wide association showed increased methylation of transcription factors involved in MAP kinase (RAS/RAF) signaling upon simultaneous treatment, while it showed decreased methylation of gene sets involved in RB/E2F signaling upon single agent treatment (Online Supplementary Figure 8F). Gene expression analysis by quantitative RT-PCR validated an additive suppression of MAP kinase (ELK1, ETS1 and CCND1) and E2F signaling (E2F1, CCNA2 and CCNE1) by the combination treatment, which are involved in cell survival and proliferation. Myeloid differentiation genes (PU.1, CEBPA and GABPA) were upregulated by the combination of BAY1436032 and azacitidine, which might be due to the higher proportion of differentiated human $\mathrm{CD} 45^{+}$cells and may not be a direct effect of the drugs. (Figure 3E, Online Supplementary Figure
S9A-C). We further validated our findings at protein level in HT1080, a fibrosarcoma cell line with an endogenous heterozygous IDH1 R132C mutation. The phosphorylation of ERK1/2 and expression of its downstream target proteins ELK1 and CYCLIN D1 was decreased in cells simultaneously treated with BAY1436032 and azacitidine. Importantly, the phosphorylation of retinoblastoma ( $\mathrm{RB})$, which is necessary for E2F mediated transcription of cell cycle transition genes, was strongly decreased in cells treated with the combination of BAY1436032 and azacitidine, while E2F1 protein levels were not affected (Figure 3F). We next evaluated if targeting the MEK pathway or cyclin dependent kinases alter the proliferation of IDH1mut AML cells using using the MEK1/2 inhibitor trametinib and the CDK4/6 inhibitor abemaciclib. Trametinib 4-fold more potently inhibited colony-forming potential in IDH1 mutant compared to IDH1 wild-type patients (Figure 3G). Similarly, the CDK4/6 inhibitor abemaciclib inhibited the colony-forming potential 3-fold more potently in IDH1 mutant compared to IDH1 wild-type patients (Figure 3F). This data suggests synergy between BAY1436032 and azacitidine in induction of differentiation through transcriptional activation of myeloid differentiation genes and inhibition of proliferation and self-renewal through inhibition of MAP-kinase and $\mathrm{RB} / \mathrm{E} 2 \mathrm{~F}$ signaling.

\section{Discussion}

Here we provide preclinical evidence that the simultaneous combination of a mIDH1 inhibitor with a hypomethylating agent significantly inhibits LSC in a synergistic manner. While the phase I dose-escalation study of mIDH1 inhibitor ivosidenib has shown encouraging results, 79\% of IDH1 mutant AML patients who had a complete remission or complete remission with partial hematologic recovery had detectable allele burden of mutant IDH1, suggesting that the IDH1 mutant clone was not eradicated. ' It was shown previously that BAY 1436032 has antileukemic activity, induces differentiation and depletes LSC 100-fold during a 4-week treatment. ${ }^{10}$ However, BAY1436032 neither has anti-leukemic activity nor induces myeloid differentiation in an IDH1 wild-type AML PDX model in vivo. ${ }^{10}$ 


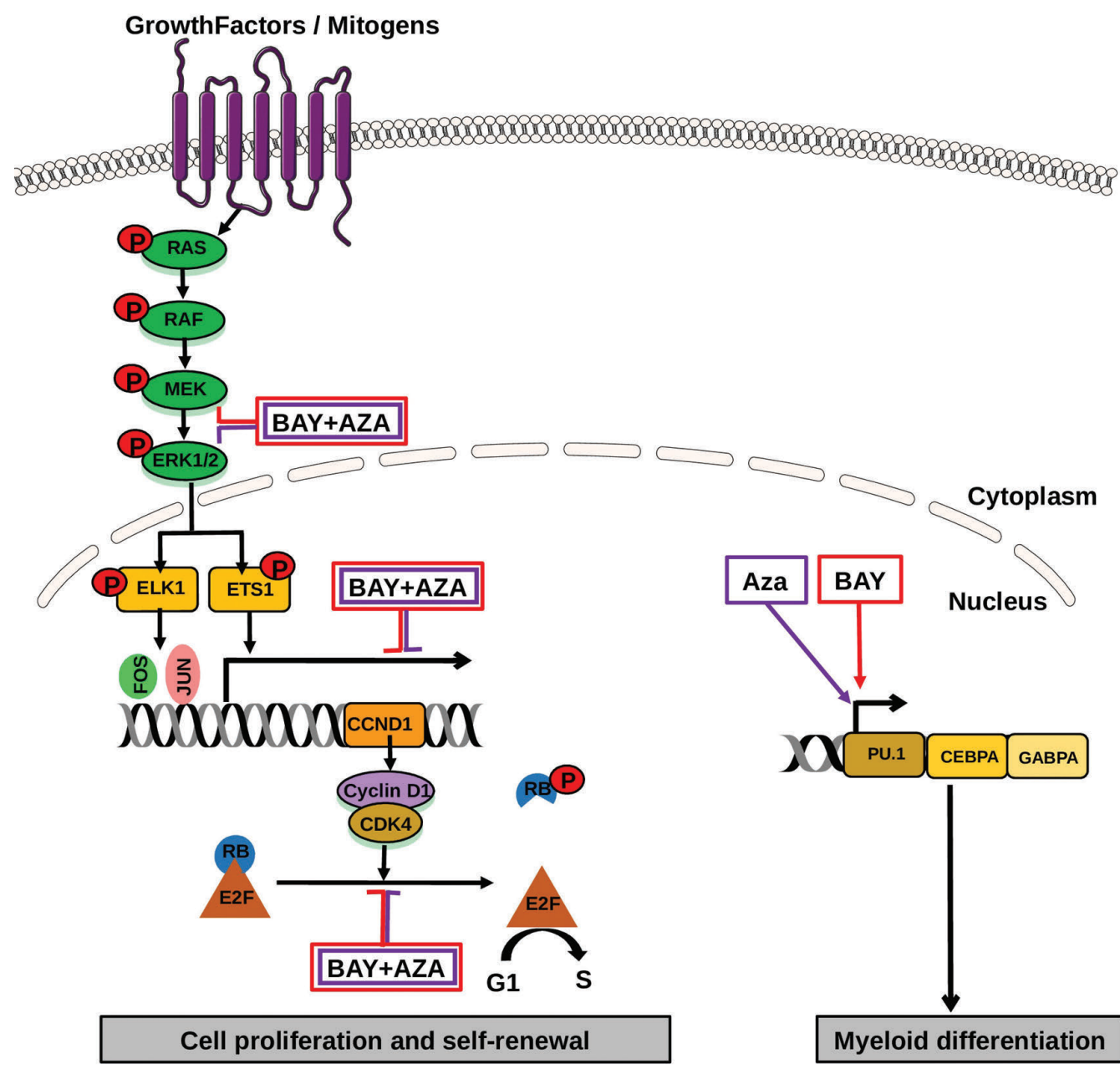

Figure 4. Model of the combined activity of BAY1436032 and azacitidine on leukemia stem cells. BAY1436032 and azacitidine (AZA) as single agents induce the expression of genes involved in myeloid differentiation (PU.1, CEBPA, and GABPA) and show additive effects in combination. MAP kinase signaling is synergistically inhibited by the combination treatment mediated by inhibition of ERK1/2 phosphorylation and suppression of its downstream targets (ELK1, ETS, CCND1).

Suppression of the RB phosphorylation by the combined treatment of BAY1436032 and azacitidine suggests synergistic inhibition of the cyclinD1/CDK4 complex. Unphosphorylated retinoblastoma binds to E2F transcription factors and prevents the $\mathrm{G} 1$ to $\mathrm{S}$ transition of the cell cycle, thereby inhibiting cell proliferation.

Azacitidine also induces differentiation and reduces selfrenewal ${ }^{15,16}$ letting us hypothesize that the combination of a mIDH1 inhibitor and azacitidine may act synergistically. Our data suggest that the combination of BAY1436032 and azacitidine has additive effects on differentiation, while it synergistically reduces LSC by 33,150-fold.

It was shown previously that mutant IDH1 activates MAPK/ERK signaling in leukemia and glioma cells. ${ }^{5,17}$ Therefore, we expected that a $\mathrm{MIDH} 1$ inhibitor would be able to reduce MAPK/ERK signaling. Data from pancreatic cancer cells suggested that treatment with azacitidine also inhibits MAPK/ERK signaling by promoter hypomethylation and induction of expression of DUSP6, which dephosphorylates members of the MAPK superfamily. ${ }^{18,19}$ However, the effect of azacitidine on MAPK signaling in leukemia patients is not known. We found that inhibition of the MAP kinase pathway is not a direct consequence of DNA hypomethylation of MAP kinase genes, but rather a transcriptional or posttranscriptional effect on genes that regulate the MAP kinase pathway.

We evaluated the consequences of MAPK/ERK inhibition and found that reduced phosphorylation of ERK1/2 leads to suppression of cyclin D1, which is then unavailable to bind CDK4. CDK4 in turn cannot phosphorylate $\mathrm{Rb}$ and consequently phosphorylation of $\mathrm{Rb}$ is strongly inhibited by the combined treatment of BAY1436032 and azacitidine, providing a mechanistic basis for reduced proliferation and self-renewal of AML cells (Figure 4).
Phosphorylation of Serine 795 of $\mathrm{Rb}$ is required for the dissociation of E2F1 from Rb, so that it can bind to DNA and induce transcription of genes involved in the G1 to S transition. ${ }^{20}$ Similarly, the phosphorylation of Serine 807 and 811 is required to prime phosphorylation at other sites on $\mathrm{Rb}{ }^{21} \mathrm{E} 2 \mathrm{~F} 1$ then remains bound to $\mathrm{Rb}$ and is unavailable for cell cycle progression, leading to the observed strong antileukemic effects.

NRAS mutations activate MAPK/ERK signaling and have been associated with resistance to $\mathrm{MIDH} 2$ inhibitor treatment. $^{22,23}$ Combination treatment with a mIDH1/2 inhibitor with azacitidine and inhibition of MAPK/ERK signaling may indeed target a specific dependency in IDH mutated leukemias and may reduce the risk of relapse.

Although IDH1/2 mutations increase DNA methylation through the oncometabolite $\mathrm{R}-2 \mathrm{HG},{ }^{24} \mathrm{IDH} 1 / 2$ mutated leukemias were not associated with better response to azacitidine monotherapy in myelodysplastic syndrome and AML patients. ${ }^{25,26}$ We conclude that the synergistic effect of BAY1436032 and azacitidine derives from specific inhibition of MAPK/ERK and RB/E2F signaling. This is supported by the striking difference between sequential and simultaneous treatment with BAY1436032 and azacitidine. Simultaneous treatment during 5 days was 70 -fold more efficient in eliminating LSC than sequential treatment, suggesting improved efficacy of the combination when both drugs are present in the leukemic cell at the same time. This strongly argues for the concurrent application of both 
drugs in ongoing and future clinical trials. A phase I clinical study combining the IDH1 inhibitor ivosidenib with azacitidine is ongoing and initial data showed promising results. ${ }^{27}$ It may be worth investigating the effect of prolonged administration of azacitidine at a lower dose or with the oral formulation CC-486 concurrently with a $\mathrm{mIDH} 1 / 2$-inhibitor to further enhance its efficacy in IDH mutated AML patients.

In conclusion, the combination of BAY1436032 and azacitidine shows a strong reduction of LSC by suppressing cell proliferation, self renewal and by inducing differentiation.

\section{Disclosures}

Chaturvedi and $M$. Heuser received research support to their University from Bayer AG. S. Kaulfuss, and M. Jeffers are employees of Bayer AG. M. Wagner, O. Panknin, and A. Haegebarth are employees and equity owners of Bayer AG. All other authors have no conflict of interest.

\section{Contributions}

A.C. and M.H. conceived and designed the study. A.C., C.G., N.B., R.Go., K.G., R.S., B.O., J.W., and R.Ge. collected the data. A.C., C.G., R.Gab., and M.H. analyzed and assembled the data. S.K., O.P., M.W., M.J., and A.H. provided critical reagents. F.T., A.G. and M.H. collected patient samples and provided the patient data. A.C. and M.H. wrote the manuscript. All authors reviewed the data and edited and approved the final version of the manuscript.

\section{Acknowledgments}

We acknowledge the assistance of the Cell Sorting Core Facility of Hannover Medical School supported in part by the Braukmann-Wittenberg-Herz-Stiftung and the Deutsche Forschungsgemeinschaft. We would like to thank all participating patients and contributing doctors, the staff of the Central Animal Facility of Hannover Medical School, and Silke Glowotz, Martin Wichmann, Annett Reinsch and Nadine Kattre for their support.

\section{Funding}

This work was supported by funding from Bayer AG, an ERC grant under the European Union's Horizon 2020 research and innovation programme (No. 638035), by grant 70112697 from Deutsche Krebshilfe; the German Federal Ministry of Education and Research grant $01 E 00802$ (IFB-TX); and DFG grants HE 5240/5-1, HE 5240/6-1 and HE5240/6-2.

\section{References}

1. Papaemmanuil E, Gerstung M, Bullinger L, et al. Genomic Classification and prognosis in acute Myeloid Leukemia. N Engl J Med. 2016;374(23):2209-2221.

2. Wagner K, Damm F, Gohring G, et al. Impact of IDH1 R132 mutations and an IDH1 single nucleotide polymorphism in cytogenetically normal acute myeloid leukemia: SNP rs11554137 is an adverse prognostic factor. J Clin Oncol. 2010; 28(14):2356-2364.

3. Dang L, White DW, Gross S, et al. Cancerassociated IDH1 mutations produce 2hydroxyglutarate. Nature. 2009; 462(7274):739-744

4. Lu C, Ward PS, Kapoor GS, et al. IDH mutation impairs histone demethylation and results in a block to cell differentiation. Nature. 2012;483(7390):474-478.

5. Chaturvedi A, Araujo Cruz MM, Jyotsana N, et al. Mutant IDH1 promotes leukemogenesis in vivo and can be specifically targeted in human AML. Blood. 2013;122(16):28772887.

6. Chaturvedi A, Araujo Cruz MM, Jyotsana N, et al. Enantiomer-specific and paracrine leukemogenicity of mutant IDH metabolite 2-hydroxyglutarate. Leukemia. 2016;30(8): 1708-1715.

7. DiNardo CD, Stein EM, de Botton $S$, et al. Durable remissions with ivosidenib in IDH1mutated relapsed or refractory AML. N Engl J Med. 2018:378(25):2386-2398.

8. Hollenbach PW, Nguyen AN, Brady H, et al. A comparison of azacitidine and decitabine activities in acute myeloid leukemia cell lines. PLoS One. 2010;5(2):e9001.

9. Dombret H, Seymour JF, Butrym A, et al. International phase 3 study of azacitidine vs conventional care regimens in older patients with newly diagnosed AML with $>30 \%$ blasts. Blood. 2015;126(3):291-299

10. Chaturvedi A, Herbst L, Pusch S, et al. Panmutant-IDH1 inhibitor BAY1436032 is highly effective against human IDH1 mutant acute myeloid leukemia in vivo. Leukemia. 2017;31(10):2020-2028.

11. Chou TC. Drug combination studies and their synergy quantification using the ChouTalalay method. Cancer Res. 2010; 70(2):440 446.

12. Chou TC. Theoretical basis, experimenta design, and computerized simulation of synergism and antagonism in drug combination studies. Pharmacol Rev. 2006;58(3):621-681.

13. Heuser M, Argiropoulos B, Kuchenbauer F, et al. MN1 overexpression induces acute myeloid leukemia in mice and predicts ATRA resistance in patients with AML. Blood. 2007;110(5):1639-1647.

14. Ng SW, Mitchell A, Kennedy JA, et al. A $17-$ gene stemness score for rapid determination of risk in acute leukaemia. Nature. 2016;540(7633):433-437.

15. Jones PA. Altering gene expression with 5azacytidine. Cell. 1985;40(3):485-486.

16. Jones PA. Effects of 5-azacytidine and its 2 'deoxyderivative on cell differentiation and DNA methylation. Pharmacol Ther. 1985; 28(1):17-27.

17. Shibata T, Kokubu A, Miyamoto M, Sasajima Y, Yamazaki N. Mutant IDH1 confers an in vivo growth in a melanoma cell line with BRAF mutation. Am J Pathol. 2011; 178(3):1395-1402

18. Xu S, Furukawa T, Kanai N, Sunamura M, Horii A. Abrogation of DUSP6 by hypermethylation in human pancreatic cancer. J Hum Genet. 2005;50(4):159-167.

19. Furukawa T, Tanii E, Xu S, Horii A. Feedback regulation of DUSP6 transcription responding to MAPK1 via ETS2 in human cells. Biochem Biophys Res Commun. 2008; 377(1):317-320. 20. Rubin SM, Gall AL, Zheng N, Pavletich NP.
Structure of the Rb C-terminal domain bound to E2F1-DP1: a mechanism for phosphorylation-induced E2F release. Cell. 2005;123(6): 1093-1106.

21. Driscoll B, T'Ang A, Hu YH, et al. Discovery of a regulatory motif that controls the exposure of specific upstream cyclin-dependent kinase sites that determine both conformation and growth suppressing activity of $\mathrm{pRb}$. J Biol Chem. 1999;274(14):9463-9471.

22. Stein EM, DiNardo CD, Pollyea DA, et al Enasidenib in mutant IDH2 relapsed or refractory acute myeloid leukemia. Blood. 2017;130(6):722-731.

23. Amatangelo MD, Quek L, Shih A, et al Enasidenib induces acute myeloid leukemia cell differentiation to promote clinical response. Blood. 2017;130(6):732-741.

24. Figueroa ME, Abdel-Wahab O, Lu C, et al. Leukemic IDH1 and IDH2 mutations result in a hypermethylation phenotype, disrupt TET2 function, and impair hematopoietic differentiation. Cancer Cell. 2010;18(6):553 567

25. Sekeres MA, Othus M, List AF, et al Randomized phase II study of azacitidine alone or in combination with lenalidomide or with vorinostat in higher-risk myelodysplastic syndromes and chronic myelomonocytic leukemia: North American Intergroup Study SWOG S1117. J Clin Oncol. 2017; 35(24):2745-2753.

26. Dohner H, Dolnik A, Tang L, et al Cytogenetics and gene mutations influence survival in older patients with acute myeloid leukemia treated with azacitidine or conventional care. Leukemia. 2018;32(12):25462557.

27. Dinardo CD, Stein AS, Stein EM, et al Mutant IDH (mIDH) inhibitors, ivosidenib or enasidenib, with azacitidine (AZA) in patients with acute myeloid leukemia (AML). J Clin Oncol. 2018;36(15):7042-7042. 